\title{
In Situ Scanning Tunneling Microscopy of DNA-modified Gold Surfaces: Bias and Mismatch Dependence
}

\author{
Donato M. Ceres and Jacqueline K. Barton* \\ Division of Chemistry and Chemical Engineering, \\ California Institute of Technology, Pasadena, CA 91125
}

Supporting Information:

Figure S1 (next page): Series of images of a sample containing 75\% PM DNA at different surface potentials. Each image has been recorded after $3 \mathrm{~min}$ in order to attain equilibrium conditions. Tunneling current: $50 \mathrm{pA}$, potentials vs Ag wire.

By following the surface changes from $-400 \mathrm{mV}$ to $+400 \mathrm{mV}$ and vice versa, one can notice the hysterisis and reversibility, where the underlying gold surface appears and disappears at different potentials. 

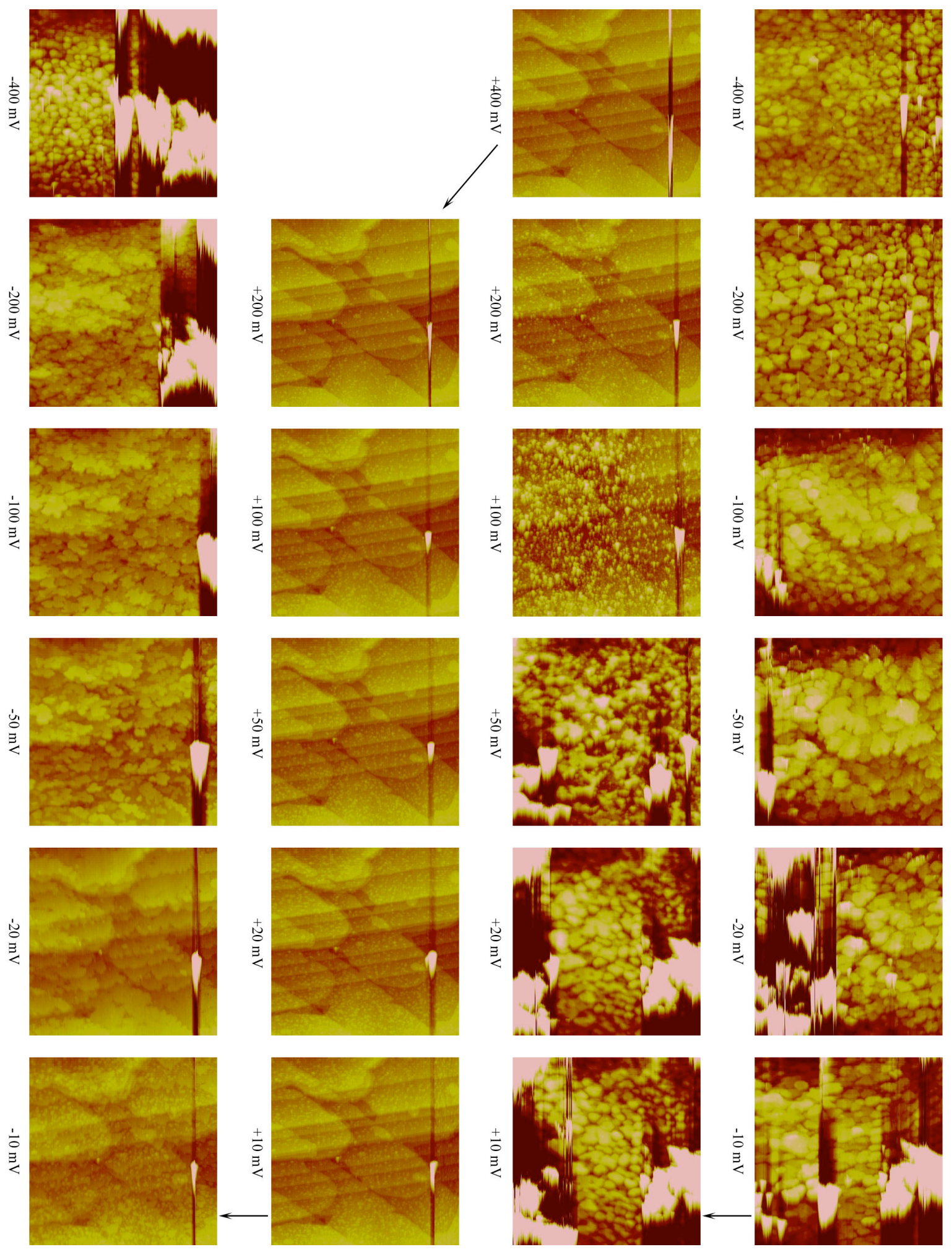

Figure S1 